\title{
Riscos ocupacionais no trabalho agrícola e a negociação para a saúde do trabalhador rural
}

Occupational risks in agricultural work and the negotiation for the health of rural workers

Riesgos laborales en el trabajo agrícola y la negociación para la salud del trabajador rural

\section{Leticia Silveira Cardoso , Clarice Alves Bonow ${ }^{\mathrm{II}}$, Daiani Modernel Xavier ${ }^{\mathrm{III}}$, Marta Regina Cezar-Vaz ${ }^{\mathrm{IV}}$}

\begin{abstract}
Resumo: Objetivo: apreender a relação entre riscos ocupacionais no trabalho agrícola e a negociação para a saúde do trabalhador rural. Método: estudo misto concorrente aninhado, combinado por integração (QUAN + QUAL) igualitária. Realizado com 351 agricultores, entre 2014/2015, por meio de formulário e entrevista semiestruturada. Para a análise, utilizou-se a estatística descritiva e inferencial e a classificação hierárquica descendente e a nuvem de palavras. Resultados: na fase QUAN, obteve-se idade média de 50,58 anos e predomínio de sintomas de adoecimento nos sistemas muscular, endócrino e urinário. Na fase QUAL, evidenciou-se que a negociação dos produtos agrícolas amplia a exposição dos trabalhadores a riscos físico, químico e ergonômico. O lucro representa o objetivo e permite o custeio de equipamentos, assistência à saúde e terapêutica medicamentosa, mediante o adoecimento. Conclusão: os riscos ocupacionais estão presentes no trabalho agrícola. A negociação reduz-se ao consumo de serviços e produtos de saúde em presença do adoecimento.
\end{abstract}

Descritores: Riscos Ocupacionais; Saúde da População Rural; Saúde do Trabalhador; Negociação; Agricultura

Abstract: Objective: to understand the relationship between occupational risks in agricultural work and the negotiation for the health of rural workers. Method: mixed nested concurrent study, combined with equal integration (QUAN + QUAL). It was conducted with 351 farmers, between 2014/2015, using a form and semistructured interview. For the analysis, descriptive and inferential statistics and the descending hierarchical classification, and the word cloud were used. Results: in the QUAN phase, the average age of 50.58 years old was obtained and a predominance of symptoms of illness in the muscular, endocrine, and urinary systems. In the QUAL phase, it became evident that the negotiation of agricultural products increases the exposure of workers to physical,

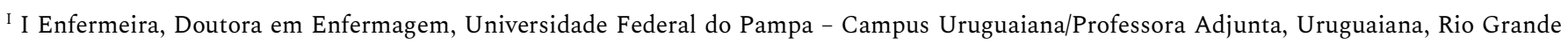
do Sul, Brasil. E-mail: 1sc_enf@yahoo.com.br. Orcid: http://orcid.org/0000-0002-2946-6758

II Enfermeira, Doutora em Enfermagem, Universidade Federal de Pelotas/Professora Adjunta, Pelotas, Rio Grande do Sul, Brasil. E-mail: claricebonow@gmail.com. Orcid: http://orcid.org/0000-0001-9918-9234

III Enfermeira, Doutora em Enfermagem, Universidade Federal do Rio Grande/Professora Adjunta, Rio Grande, Rio Grande do Sul, Brasil. Email: daiamoder@gmail.com. Orcid: http://orcid.org/0000-0003-3832-2120

IV Enfermeira, Doutora em Filosofia da Enfermagem, Universidade Federal do Rio Grande/Professora Titular, Rio Grande, Rio Grande do Sul, Brasil. E-mail: mrcezarvaz@gmail.com. Orcid: http://orcid.org/0000-0002-0754-7469
} 
Riscos ocupacionais no trabalho agrícola e a negociação para a saúde do trabalhador rural | 2

chemical, and ergonomic risks. Profit represents the goal and allows for the cost of equipment, health care, and drug therapy, through illness. Conclusion: occupational risks are present in agricultural work. Negotiation is reduced to the consumption of health services and products in the presence of illness.

Descriptors: Occupational Risks; Rural Health; Occupational Health; Negotiating; Agriculture

Resumen: Objetivo: comprender la relación entre riesgos laborales en el trabajo agrícola y la negociación para la salud del trabajador rural. Método: estudio mixto concurrente anidado, combinado por integración (QUAN + QUAL) igualitaria. Fue realizado con 351 agricultores, entre 2014/2015, por medio de un formulario y una entrevista semiestructurada. Para el análisis, se utilizó la estadística descriptiva e inferencial y la clasificación jerárquica descendiente y la nube de palabras. Resultados: en la fase QUAN, se obtuvo una edad media de 50,58 años y predominio de síntomas de enfermedades en los sistemas muscular, endócrino y urinario. En la fase QUAL, se observó que la negociación de los productos agrícolas amplia la exposición de los trabajadores a riesgos físico, químico y ergonómico. El lucro representa el objetivo y permite el costeo de equipamientos, asistencia a la salud y terapéutica medicamentosa, mediante la enfermedad. Conclusión: los riesgos laborales están presentes en el trabajo agrícola. La negociación se reduce al consumo de servicios y productos de salud en la presencia de la enfermedad.

Descriptores: Riesgos laborales; Salud rural; Salud laboral; Negociación; Agricultura

\section{Introdução}

O trabalho agrícola caracteriza-se pela manipulação humana e tecnológica da terra. A manipulação humana torna homens e mulheres mais vulneráveis à ocorrência de problemas de saúde, pelo contato direto com agentes nocivos do processo produtivo. Já a manipulação tecnológica, reduz tal contato, mas acelera o processo produtivo e impacta sobre a exposição ocupacional desses trabalhadores. A exposição é apreendida pelos próprios agricultores em diferentes níveis e ainda restrita na apropriação de conhecimentos e comportamentos de proteção e segurança. ${ }^{1}$

A restrição de conhecimentos e comportamentos de proteção e segurança no trabalho rural apresenta enorme valor para o setor socioeconômico mundial, pois em torno de 520 milhões de homens e 410 milhões de mulheres foram empregados na agricultura, a qual é a principal fonte de empregos na maioria dos países em desenvolvimento. Atualmente, o setor da agricultura responde por $60 \%$ de todos os postos de trabalho na África Subsaariana. ${ }^{2}$ No Brasil, o setor agrícola desempenha um papel significativo no desenvolvimento econômico do país, apesar da agricultura ter ocupado apenas 5,4\% do produto interno bruto em $2010-2013 .{ }^{3}$ No que 
se refere às questões sociais, a importância está relacionada à distribuição da renda e na manutenção do trabalhador no campo.

O trabalho agrícola é uma atividade que requer esforço físico e demanda alto consumo de energia humana. Muitas vezes, inadequado ao ser humano que subordinado à economia de mercado negocia o produto agrícola visando sua sobrevivência e não percebendo que os riscos presentes no ambiente de trabalho podem causar injúrias à saúde. ${ }^{4}$

A exposição às radiações solares por longos períodos pode desencadear lesões cutâneas de caráter benigno e, até mesmo, o câncer de pele. ${ }^{5}$ Os ruídos decorrentes do uso de motosserras, colheitadeiras e tratores podem resultar na diminuição ou perda progressiva da audição, fadiga, irritabilidade, elevação da pressão arterial e distúrbios do sono. ${ }^{6}$ Além disso, são frequentes os acidentes com animais peçonhentos, exposição a partículas de grãos, ácaros e pólen, os quais podem ocasionar doenças respiratórias, como a asma e as pneumonites, por hipersensibilização. ${ }^{7}$

A divisão e o ritmo intenso de trabalho na agricultura, com cobrança de produtividade e presença de problemas ergonômicos, geram riscos à saúde dos trabalhadores. ${ }^{8}$ Estes apresentam maior prevalência de morbidades com surgimento das Lesões por Esforços Repetitivos e Distúrbios Osteomusculares Relacionadas com o Trabalho (LER/DORT). ${ }^{9}$ Ao que se somam as intoxicações graves decorrentes da exposição a fertilizantes e agrotóxicos, produtos do grupo dos fosfatos, sais de potássio e nitratos, que podem ocasionar hipocalcemia, ulceração da mucosa gástrica, hemorragia e câncer. ${ }^{10}$

Adoecimentos decorrentes desse trabalho se agravam por ausência de identificação/intervenção profissional e adoção de terapêutica adequada. Ausência a ser transposta em nível de atenção primária à saúde cuja finalidade está em promover a saúde das comunidades. Promoção que deve primar pela acessibilidade aos serviços e pela execução de ações de orientação dos trabalhadores agricultores para minimização da exposição a riscos ocupacionais. ${ }^{11}$ 
Riscos ocupacionais no trabalho agrícola e a negociação para a saúde do trabalhador rural | 4

A orientação em saúde caracteriza-se como parte do cuidado da enfermagem a famílias em ambiente rural, visualizando-se que os enfermeiros dispõem de uma organização do processo de trabalho que os permite intervir a nível domiciliar/familiar a fim de usufruírem da dialogicidade interpessoal e ambiental. ${ }^{12}$ Dialogicidade esta que representa a captação de informações clínicas do conjunto de trabalhadores com fins à materialização do diagnóstico situacional. ${ }^{13}$ Este congrega os dados do processo saúde-doença e permite a enfermagem e aos demais profissionais da saúde a negociação, a organização, o planejamento e a programação de ações/intervenções em prol da mudança de hábitos inseguros. ${ }^{14}$

Nesse contexto, tem-se como questão de pesquisa: qual a relação existente entre riscos ocupacionais no trabalho agrícola e a negociação para a saúde do trabalhador rural? A partir desta, elaborou-se o presente estudo com o objetivo de apreender a relação entre riscos ocupacionais no trabalho agrícola e a negociação para a saúde do trabalhador rural.

\section{Método}

Estudo com método misto, do tipo concorrente aninhado, no qual os dados quantitativos e qualitativos são coletados concomitantemente. ${ }^{15}$ Estão mixados mediante integração (QUAN + QUAL), com atribuição igualitário de pesos. Essa integração foi planejada desde a construção dos instrumentos de coleta de dados. Tal construção sustentou-se por conectividades das variáveis utilizadas já publicitadas cientificamente em diversos estudo, por exemplo, tipo, horas de trabalho, uso de agrotóxico e distúrbios osteomusculares, baixa escolaridade, renda e proteção contra os riscos ocupacionais, bem como por experiências prévias realizadas pelo coletivo de pesquisadores do Laboratório de Processos Socioambientais e Produção Coletiva de Saúde - LAMSA, vinculado à Universidade Federal do Rio Grande (FURG). ${ }^{12,16}$ Teoricamente, alicerça-se na integralidade da atenção à saúde, especificamente pela compreensão dos nexos causais estabelecidos entre o trabalho rural e o adoecimento do trabalhador. ${ }^{14}$ Justifica-se a 
tipificação do método a partir do processo de seleção da amostra e de acesso ao cenário e participantes da pesquisa.

O cenário da pesquisa constituiu-se por dois municípios do estado do Rio Grande do Sul (RS), o situado na região sul apresenta uma área territorial de $2.709,391 \mathrm{~km}^{2}$; com densidade demográfica de 72,79 hab $/ \mathrm{km}^{2}$; índice de desenvolvimento humano municipal (IDHM) de 0,744 e ocupa o 35ํㅣㅁ lugar no ranking estadual para atividade econômica de agropecuária. Já o da região oeste possui, respectivamente, $5.702,098 \mathrm{~km}^{2} ; 21,95 \mathrm{hab} / \mathrm{km}^{2} ;$ o mesmo IDHM, mas ocupa o $4^{\circ}$ lugar no referido ranking. ${ }^{17}$ Tais municípios localizam-se a uma distância de $618 \mathrm{~km}$, considerando-se o deslocamento por rodovia, BR 293, percorrido pelo conjunto de oito pesquisadores que realizaram as etapas de coleta de dados em dupla.

A população da pesquisa, etapas quantitativa e qualitativa, constituiu-se de 371 trabalhadores agricultores, definidos como pessoas responsáveis pelo gerenciamento da produção primária de frutas, hortaliças, grãos e outros produtos agrícolas. O computo da população ocorreu pela sobreposição de dados do Instituto Brasileiro de Geografia e Estatística (IBGE) do ano de 2010, de Sindicatos dos Trabalhadores Rurais; das Empresas de Assistência Técnica e Extensão Rural (EMATER) e da Secretaria Municipal de Agricultura, do ano de 2013. Essa sobreposição foi realizada para se obter somente as informações dos trabalhadores da agricultura, excluindo-se os da pecuária, produção florestal, pesca e aquicultura.

Dessa população foram decrescidos 24 agricultores que não atendiam aos critérios de seleção pré-estabelecidos - indivíduos maiores de 18 anos, residentes em ambiente rural e, que atuassem diretamente no trabalho da agricultura de hortifrutigranjeiros. Portanto, excluíram-se quatro agricultores por serem menores de 18 anos de idade; 12 por não residirem no ambiente rural, e oito agricultores por não atuarem diretamente no trabalho da agricultura de hortifrutigranjeiros. Para o quantitativo de 347 agricultores, foram aplicados os seguintes critérios de exclusão: não consentir a entrevista audiogravada e impossibilidade de acesso ao 
Riscos ocupacionais no trabalho agrícola e a negociação para a saúde do trabalhador rural | 6

domicílio. Neste conjunto, houve a diminuição de 16 participantes, dos quais 10 não se encontravam no domicílio nas três tentativas de contato realizadas por meio de visita domiciliar de uma dupla de pesquisadores em companhia de um trabalhador da EMATER e seis que não consentiram a realização da coleta de dados. O número de participantes ficou constituído de 331 participantes no presente estudo.

A coleta de dados com os agricultores iniciou-se pela região sul em março de 2014, em consecutivo pela oeste, sendo concluída em julho de 2015. A apresentação dos pesquisadores aos participantes foi realizada pelo trabalhador da EMATER. A amostra do estudo compôs-se por 331 trabalhadores agricultores que atenderam aos referidos critérios e consentiram a realização da pesquisa após apresentação da proposta.

Para a coleta de dados denominada como fase 1, QUAN, utilizou-se como instrumento um formulário com variáveis quantitativas discreta (exemplo: idade) e contínua (exemplo: renda) referentes aos dados de identificação do participante e ao número de agravos à saúde por sistema do organismo humano presentes no período de 2013 a 2015. E com variáveis qualitativas/categóricas nominal (exemplo: estado civil, tipo de agravo, produto da negociação, participantes) e ordinal (exemplo: escolaridade) referentes à complementação dos dados de identificação, tipo de agravos à saúde por sistema do organismo humano no período supracitado e à frequência da procura por assistência à saúde.

Para a análise do conjunto das variáveis, utilizou-se o software Statistical Package for the Social Sciences (versão 21.0, SPSS, Inc., Chicago, IL, USA), aplicou-se a estatística descritiva cujos dados foram apresentados por frequência absoluta e relativa e de dispersão (média e desvio padrão). E realizou-se o teste qui-quadrado de Pearson, utilizando-se com nível de significância estatística p $\geq 0,05$ para a análise inferencial. ${ }^{18} \mathrm{Na}$ fase 2, QUAL, realizou-se entrevista semiestruturada audiogravada a partir de um roteiro previamente elaborado. Houve uma perda de 126 participantes, resultando em uma amostra de 205 trabalhadores agricultores que mantiveram o interesse em 
participar e consentiram a audiogravação e o acesso ao interior de seu domicílio. As entrevistas ocorreram ao término do preenchimento do formulário da fase 1, geralmente na sala ou na cozinha do domicílio, sem a presença de outros membros familiares em fase adulta.

As entrevistas duraram de 33 a 67 minutos, foram digitalizadas e organizadas em um banco de dados no formato de texto. Para este estudo, selecionou-se a seguinte questão do roteiro da pesquisa: como a negociação no trabalho melhora a saúde dos agricultores? Dê exemplo. As respostas foram transformadas em um único corpus textual, conforme orientações prévias do software de análise Interface de $R$ pour les Analyses Multidimensionnelles de Textes et de Questionnaires (Iramuteq). E codificadas, de acordo com o Quadro 1, no qual a primeira numeração representa o número do participante; a segunda, a região da pesquisa; e a terceira, o sexo do participante.

Quadro 1 - Códigos utilizados no software IRAMUTEQ.

\begin{tabular}{|c|c|c|}
\hline Participante & Posição & Unidade \\
\hline De *n_01 a *n_205 & $\begin{array}{c}\text { *posic_1: sul do RS } \\
\text { *posic_2: oeste do RS }\end{array}$ & ${ }^{*}$ "uni_1: Feminino \\
*uni_2: Masculino \\
\hline
\end{tabular}

Para a análise do corpus textual, aplicou-se a Classificação Hierárquica Descendente (CHD) e a análise de nuvem de palavras. A primeira permite a identificação da quantidade de palavras, da frequência média e do número de hapax (palavras com frequência um), resultando em um dendograma. A segunda utiliza a frequência de ocorrência das palavras para agrupá-las e organizá-las graficamente. ${ }^{19}$

Os dados analisados têm origem no projeto de pesquisa intitulado: "Natureza humana da força de trabalho masculina e feminina: um estudo com trabalhadores(as) em dois ambientes rurais do Rio Grande do Sul”, aprovado em março de 2013 pelo Comitê de Ética em Pesquisa, protocolo $\mathrm{n}^{\mathbf{0}}: 23116.000645 / 2013-73$. Estão em consonância com as diretrizes e normas regulamentadoras da Resolução 466/2012 do Conselho Nacional de Saúde/MS sobre Pesquisas 
Riscos ocupacionais no trabalho agrícola e a negociação para a saúde do trabalhador rural | 8

Envolvendo Seres Humanos. O anonimato dos participantes, bem como a possibilidade de retirar o consentimento ou solicitar esclarecimentos sobre a pesquisa, foi comunicado e disponibilizado no Termo de Consentimento Livre e Esclarecido do participante, assinado previamente no início da coleta de dados, em duas vias. Para manutenção desse anonimato, ao apresentar-se os resultados deste estudo, utilizou-se a codificação expressa no Quadro 1.

\section{Resultados}

A amostra da fase QUAN incluiu agricultores com idade média de 50,58 anos $(\mathrm{DP} \pm 13,97)$ e renda mensal familiar média de $\mathrm{R} \$ 1.827,97(\mathrm{DP} \pm \mathrm{R} \$ 2.181,25)$. Na região sul do $\mathrm{RS}$ participaram $201(60,7 \%)$ agricultores, sendo a maioria do sexo feminino ( $\mathrm{n}=122 ; 60,7 \%)$. Já na região oeste, entre os $130(39,3 \%)$ participantes, predominou o sexo masculino ( $\mathrm{n}=74 ; 56,2 \%)$. A Tabela 1 apresenta os demais dados de identificação dos participantes.

Tabela 1 - Dados de identificação dos trabalhadores agricultores ( $\mathrm{n}=331)$ - RS, 2015.

\begin{tabular}{llcc}
\hline Variáveis & \multicolumn{1}{c}{ Categorização } & Frequência & \% \\
\hline \multirow{2}{*}{ Localidade } & Sul & 201 & 60,7 \\
& Oeste & 130 & 39,3 \\
& Feminino & 182 & 55,0 \\
Cor da pele & Masculino & 149 & 45,0 \\
& Branca & 304 & 91,8 \\
& Negra & 8 & 2,4 \\
& Parda & 18 & 5,4 \\
Estado civil & Indígena & 1 & 0,3 \\
& Casados & 273 & 82,5 \\
& Solteiros & 36 & 10,9 \\
& Viúvos & 11 & 3,3 \\
& Separados & 11 & 3,3 \\
& Não alfabetizados & 23 & 6,9 \\
& Ensino fundamental incompleto & 220 & 66,5 \\
& Ensino fundamental completo & 32 & 9,7 \\
\multirow{5}{*}{ Escolaridade } & Ensino médio incompleto & 19 & 5,7 \\
& Ensino médio completo & 25 & 7,6 \\
& Curso técnico & 4 & 1,2 \\
& Superior incompleto & 05 & 1,5 \\
& Superior completo & 02 & 0,6 \\
& Pós-graduação completa & 01 & 0,3 \\
\hline
\end{tabular}


A procura anual pelos serviços de saúde apresentou grande frequência entre os trabalhadores de ambas as regiões, destacando-se maior referência por parte das agricultoras. Paralelamente, tem-se uma maior proporção de trabalhadores da região oeste, comparativamente à região sul, que não procura os serviços de saúde (n=31;23,8\%). Os demais dados são apresentados na Tabela 2.

Tabela 2 - A procura dos agricultores pelos serviços de saúde - RS, 2015.

\begin{tabular}{lcccccccc}
\hline Variáveis & \multicolumn{2}{c}{ Sul } & \multicolumn{2}{c}{ Oeste } & \multicolumn{2}{c}{ Feminino } & \multicolumn{2}{c}{ Masculino } \\
& $\mathrm{n}(\mathbf{2 0 1})$ & $\mathbf{\%}$ & $\mathrm{n}(\mathbf{1 3 0})$ & $\mathbf{\%}$ & $\mathbf{n}(\mathbf{1 8 2})$ & $\mathbf{\%}$ & $\mathbf{n}(\mathbf{1 4 9 )}$ & $\%$ \\
\hline Não procuram & 15 & 7,5 & 31 & 23,8 & 12 & 6,6 & 34 & 22,8 \\
Bimestralmente & 17 & 8,4 & 5 & 3,8 & 12 & 6,6 & 10 & 6,7 \\
Semestralmente & 33 & 16,4 & 11 & 8,5 & 40 & 22,0 & 4 & 2,7 \\
Anualmente & 62 & 30,8 & 45 & 34,6 & 60 & 33,0 & 47 & 31,5 \\
Bianualmente & 12 & 6,0 & 14 & 10,8 & 12 & 6,6 & 14 & 9,4 \\
Trianualmente & 5 & 2,5 & 3 & 2,3 & 6 & 3,3 & 2 & 1,3 \\
Outro & 57 & 28,4 & 21 & 16,2 & 40 & 21,9 & 38 & 25,6 \\
\hline
\end{tabular}

Sinais e sintomas de adoecimento no último ano foram autorreferenciados por 255 (77,0\%) agricultores. Os principais sistemas do organismo humano acometidos estão dispostos na Tabela 3.

Tabela 3 - Sistemas com sinais e sintomas manifestados pelos agricultores - RS, 2015.

\begin{tabular}{|c|c|c|c|c|c|c|c|c|c|c|}
\hline \multirow[t]{2}{*}{ Sistemas } & \multicolumn{2}{|c|}{ Sul } & \multicolumn{2}{|c|}{ Oeste } & \multirow[t]{2}{*}{$\mathrm{p}^{*}$} & \multicolumn{2}{|c|}{ Feminino } & \multicolumn{2}{|c|}{ Masculino } & \multirow[t]{2}{*}{$\mathrm{p}^{*}$} \\
\hline & $\begin{array}{c}\mathrm{n} \\
(201)\end{array}$ & $\%$ & $\begin{array}{c}\mathrm{n} \\
(130)\end{array}$ & $\%$ & & $\begin{array}{c}n \\
(182)\end{array}$ & $\%$ & $\begin{array}{c}\text { n } \\
(149)\end{array}$ & $\%$ & \\
\hline Articular & 65 & 32,3 & 55 & 42,3 & 0,065 & 73 & 40,1 & 47 & 31,5 & 0,107 \\
\hline Nervoso & 11 & 5,5 & 9 & 6,9 & 0,589 & 10 & 5,5 & 10 & 6,7 & 0,644 \\
\hline Cardiovascular & 14 & 7,0 & 9 & 6,9 & 0,988 & 14 & 7,7 & 9 & 6,0 & 0,556 \\
\hline Digestório & 14 & 7,0 & 6 & 4,6 & 0,381 & 11 & 6,0 & 9 & 6,0 & 0,999 \\
\hline Respiratório & 15 & 7,5 & 4 & 3,1 & 0,094 & 12 & 6,6 & 7 & 4,7 & 0,461 \\
\hline Tegumentar & 12 & 6,0 & 1 & 0,8 & 0,017 & 6 & 3,3 & 7 & 4,7 & 0,514 \\
\hline Urinário & 8 & 4,0 & 4 & 3,1 & 0,668 & 10 & 5,5 & 2 & 1,3 & 0,044 \\
\hline Muscular & 13 & 6,0 & 4 & 3,1 & 0,172 & 14 & 7,7 & 3 & 2,0 & 0,020 \\
\hline Esquelético & 3 & 1,5 & 1 & 0,8 & 0,556 & 4 & 2,2 & 00 & 0,0 & 0,069 \\
\hline Endócrino & 3 & 1,5 & 2 & 1,5 & 0,973 & 5 & 2,7 & 00 & 0,0 & 0,041 \\
\hline Genital & 1 & 0,5 & 0 & 0,0 & 0,421 & 1 & 0,5 & 00 & 0,0 & 0,365 \\
\hline Linfático & 1 & 0,5 & 0 & 0,0 & 0,421 & 1 & 0,5 & 00 & 0,0 & 0,365 \\
\hline
\end{tabular}

* teste qui-quadrado de Pearson com nível de significância estatística $p \geq 0,05$ 
Riscos ocupacionais no trabalho agrícola e a negociação para a saúde do trabalhador rural | 10

A maioria dos agricultores das regiões sul e oeste indicou com maior frequência sintomas no sistema articular (32,3\% e 42,3\%, respectivamente). Quanto ao teste estatístico, pode-se inferir que a sintomatologia associada ao sistema tegumentar apresentou significância estatística, o que ratifica hipótese de que o trabalho rural na agricultura pode provocar lesões de pele. Dos 13 agricultores que as indicam, nove manifestaram lesões com alteração na coloração da pele; dois com coleção de líquidos e outros dois com formação de sólido.

$\mathrm{Na}$ análise por sexo, os sinais e sintomas com significância estatística, associam-se respectivamente, aos sistemas muscular, endócrino e urinário. Para o sistema muscular, houve a manifestação de 17 agricultores, em que as sintomatologias estiveram mais frequentemente presentes entre as agricultoras, referenciadas a partir de dores em ligamentos e pela infecção de tendões e fáscias dos músculos de membros superiores e inferiores. Já a contração muscular (câimbra) de membros inferiores foi autorreferida pelos homens. Para o sistema endócrino, tevese a manifestação de irregularidades do ciclo menstrual por cinco agricultoras. Para o sistema urinário, houve a manifestação de 12 agricultores, em que dor, desconforto e ardência à micção estiveram presentes no sexo feminino e redução do jato e obstrução do fluxo no masculino.

Com relação aos produtos negociados, 259 (78,2\%) trabalhadores destacaram as sementes, $245(74 \%)$ os adubos e $218(65,8 \%)$ o fornecimento de produtos. Eles indicaram os comerciantes $(\mathrm{n}=149 ; 45 \%)$ como principais consumidores da produção agrícola, seguidos pelos próprios consumidores $(n=116 ; 35 \%)$ e pelos revendedores, ou seja, pessoas que negociam os produtos com os agricultores e os compradores ( $\mathrm{n}=114 ; 34,4 \%)$.

A aplicação do método de CHD, fase QUAL ( $n=205)$, resultou em 5 classes semânticas distintas. A classe 1, correspondente a $17,1 \%$ do corpus, e demonstrou com quem ocorre a negociação no trabalho agrícola. Pode-se perceber que na região sul as agricultoras referem fazê-la diretamente com os revendedores $(n=60)$ e com os consumidores diretos (familiares e vizinhos, por exemplo) ( $\mathrm{n}=87$ ). Enquanto os agricultores somente com revendedores. Ambos a 
indicam como benéfica para o aumento do lucro por possibilitar jornadas de trabalho mais longas no cultivo e colheita da produção. Paralelamente, revelaram a relação com danos à saúde dos trabalhadores que sentem dores e se expõem a riscos ocupacionais, físicos e ergonômicos.

Eu mesma negocio porque o que eu produzo não tenho compromisso com supermercado, [...] com ninguém [...], quando levo 500 pés de alface para o hortifrutigranjeiro, vende bem, volto toda influída para casa para trabalhar, mesmo que sinta dores nas costas [...]. (n_121; posic_1; uni_1)

[...] A melhoria do trabalho agrícola está em realizar com ela [revendedora] [...] eu dou pronto, ela leva e vende. Isso mudou, melhorou porque eu não tenho aquela preocupação de pegar minhas plantas e levar para vender [...] eu só entrego e consigo ficar trabalhando, por mais tempo na lavoura, mesmo sabendo que fico exposto ao sol[...]. (n_91; posic_2; uni_2)

A classe 2 , correspondente a $26,4 \%$ do corpus, associa-se diretamente à classe 3 . A primeira revela quem realiza a negociação e a segunda aponta para os objetivos desta. Em ambas as regiões se verifica que a realização da negociação ocorre pelos integrantes da família, especialmente os cônjuges. Isso permite a adequação do cultivo às exigências e necessidades dos consumidores/revendedores, entre as quais se tem a redução dos riscos químicos decorrentes da utilização de agrotóxicos.

Meu marido e eu negociamos tudo, a quanto vamos vender, se vamos fazer promoção [...] é uma negociação familiar [...] no dia da promoção aumenta muito mais o consumo, muda todo o meu trabalho, trabalho mais [...] não para a minha saúde. (n_130; posic_1; uni_1)

Porque nossa agricultura é familiar, tem que ser nós mesmos para fazer, não teria como uma terceira pessoa fazer, nós sabemos o que produzimos, temos como ter um controle do que nós produzimos, do que vamos vender. Essa negociação tem mudanças, estamos fazendo um contrato com o mercado [...] se nós precisarmos aumentar a nossa produção [...] plantar algum outro produto ou se temos que tirar fora algum produto que tenhamos [...]. É isso a negociação visando a saúde, o pouco que fazemos é usar menos agrotóxicos na plantação.(n_259; posic_2; uni_2) 
Riscos ocupacionais no trabalho agrícola e a negociação para a saúde do trabalhador rural | 12

A classe 3 , correspondente a $14,7 \%$ do corpus, associa-se diretamente à classe 2 . Ela demonstra qual o objetivo dos agricultores durante a realização da negociação da produção agrícola. Pode-se observar que eles não se preocupam diretamente com sua saúde, e sim com sua sobrevivência e lucro. A partir deste, tem-se a redução de esforços físicos aplicados ao processo produtivo devido à aquisição de equipamentos.

Sou eu quem negocia os interesses da família para comprar, vender e o objetivo é de sobreviver aqui. Mudanças só os lucros, os benefícios, compro alguma coisa para trabalha. Sobre a saúde do trabalhador não tem nenhum [agricultor] que pense. (n_129; posic_1; uni_2)

Eu e meu irmão negociamos para vender, pegar um melhor resultado, melhor preço. Mudança é que o cara [agricultor] se torna mais responsável, mais cuidadoso para negociar e ter um melhor lucro. Na saúde, o cara [agricultor] busca vender coisa melhor, para ter um melhor preço, para ter mais dinheiro para comprar equipamento, para melhorar, para diminuir o esforço físico [...]. Geralmente, o cara [agricultor] não vê a saúde, vê o lucro, que vem [...] e vai melhorar tudo. (n_294; posic_2; uni_2)

A classe 4 , correspondente a $22 \%$ do corpus, associa-se diretamente à classe 1 . Nela, tem-se a identificação do que é negociado e como a venda dos produtos agrícolas promove a saúde dos trabalhadores. Para alguns trabalhadores, o lucro advindo da negociação dos produtos torna-se importante por poder custear o tratamento de comorbidades associadas ao trabalho na agricultura.

[...] A negociação visando à saúde do trabalhador ocorre vendendo as mercadorias, como cebola e tomate para ter o dinheiro para manter a saúde [...]. (n_111; posic_1; uni_1)

Temos dores na coluna. Nós lutamos para ganhar dinheiro para conseguir comprar remédios para manter a saúde [...] ganhamos eles [remédios] no posto de saúde [...] mas têm remédios muito caros, às vezes, não nos dão e temos que comprar. (n_309; posic_2; uni_1)

A classe 5, correspondente a $19,9 \%$ do corpus, está diretamente relacionada com as classes 2 e 3. Nesta constata-se que alguns trabalhadores são capazes de perceber prejuízos à 
saúde em decorrência de hábitos e costumes, a exemplo do consumo de bebidas alcóolicas. Além disso, o desconhecimento de uma possível relação entre a negociação do trabalho agrícola com a saúde mostra certo descomprometimento com a mesma em razão da facilidade de acesso a postos de saúde aos quais recorrem ao atendimento apenas quando estão doentes.

[...] Não tem negociação para a saúde do trabalhador. Não tem nenhuma. Não, porque o posto está ali para atender quando os agricultores estão doentes[...]. (n_12; posic_1; uni_2)

[...] Tens que te cuidar, trabalhar certo, se alimentar bem, não beber, não andar bêbado porque o que estraga muito a saúde do agricultor é a bebida [...] mesmo sabendo de tudo isso, bebo de vez enquanto nos intervalos do trabalho. (n_245; posic_2; uni_2)

Verificou-se que os agricultores negociam para vender seus produtos, quais sejam: cebola, beterraba, cenoura, entre outros. Também compram os insumos necessários à realização e manutenção do plantio/plantação. A negociação não é identificada pelos agricultores como benéfica à sua saúde, visto que é realizada em prol do lucro que garante a sua a subsistência e sobrevivência. Eles se sujeitam à exposição a agentes físicos, como o sol e químicos, como os agrotóxicos, durante o trabalho, o pode gerar impactos negativos a sua saúde.

A nuvem de palavras agrupa e organiza as palavras graficamente em função da sua frequência. É uma análise lexical mais simples, porém graficamente interessante, na medida em que possibilita a rápida identificação das palavras-chave do corpus, conforme Figura 1. 


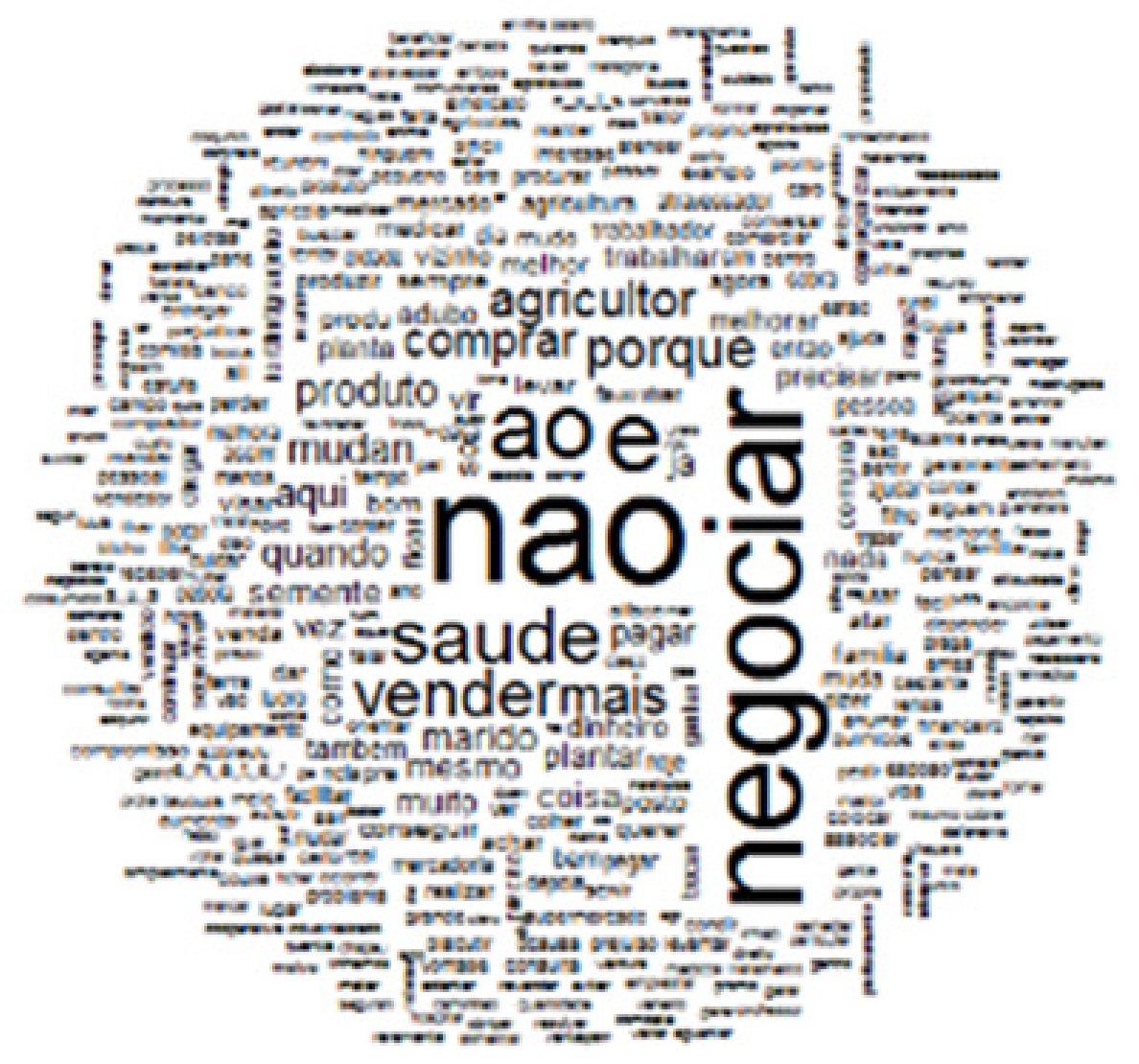

Figura 1 - Nuvem de palavras.

Por meio do método de nuvem de palavras evidencia-se que o agricultor não negocia para ter saúde, e sim para vender seus produtos. Os agricultores identificam que a negociação com vista na venda de seus produtos é necessária para a produção de novas plantações e para continuar a sobreviver do trabalho agrícola.

\section{Discussão}

Os resultados fornecem informações sobre a saúde dos trabalhadores agricultores e sugerem que a intensidade do trabalho de produção de hortifrutigranjeiros os expõe a riscos ocupacionais. E que a negociação dos produtos desse trabalho viabiliza a assistência à sua saúde quando em presença de agravos estabelecidos. 
Estudo japonês apontou a mesma postura desses trabalhadores em países orientais, justificada por aspectos relativos aos meios de transporte e à distância geográfica entre o ambiente de produção e os serviços de assistência à saúde. ${ }^{20}$ Estudo brasileiro realizado com agricultores do extremo sul do RS revela que eles se sentem satisfeitos com o tipo de trabalho, com a autonomia e com a condição de sustentar a família a partir dos resultados do trabalho. Paralelamente, identificam impactos negativos decorrentes da ausência de suporte técnico para o cultivo e para o uso de agrotóxicos, o que ocasiona danos ao ambiente e à saúde dos trabalhadores na região. ${ }^{21}$

Sintomas como irritação da pele e dos olhos, dor de cabeça e tonturas estão presentes em 34\% de 318 produtores de algodão de Punjab, Paquistão. Tais sintomas indicam o alto nível de exposição dos trabalhadores a riscos químicos, por exemplo, a utilização de agrotóxicos, que são entendidos como comuns pelos agricultores. Isso gera uma baixa procura destes pelos serviços de saúde ou mesmo por atendimento profissional. ${ }^{22}$

Insuficiência de informações e conhecimentos a respeito da saúde e da segurança, especialmente para o uso de equipamentos de proteção individual por trabalhadores rurais, foi associada à sua baixa escolaridade. Condição que também se constituiu como fator causal para intoxicações de agricultores do sexo masculino, no estado brasileiro de Minas Gerais. ${ }^{23}$ Acredita-se que ela pode também estar contribuindo para minimizar a negociação no tocante à produção de benefícios para a melhoria da saúde dos trabalhadores agricultores. Uma vez que, se tem a presença, com significância estatística, de sinais e sintomas tegumentares, musculares e urinários e ainda assim predomina a busca anual pelos serviços de saúde por parte dos participantes deste estudo.

As lesões com alteração na coloração da pele computadas na fase QUAN elucidam-se nas classes 1 e 3 da CHD e na nuvem de palavras. As atividades femininas de venda dos produtos agrícolas no hortifrutigranjeiro e as masculinas de cultivo da terra são realizadas em ambientes com exposição à radiação natural ultravioleta. Esta tem determinado maior probabilidade de lesões tegumentares na região da face de trabalhadores rurais, que ao serem comparados com 
Riscos ocupacionais no trabalho agrícola e a negociação para a saúde do trabalhador rural | 16

outros não rurais encontram-se em uma faixa etária significativamente mais jovem. ${ }^{6}$ Estudo realizado na Escócia com população rural diagnosticada com melanoma revelou que o tratamento com excisão da lesão de pele ocorre predominantemente na atenção primária, o que indica o acesso dessa população aos serviços de saúde e fortalece a presença de alterações no sistema tegumentar para tal busca pelos serviços de saúde. ${ }^{24}$

No contexto brasileiro, o acesso à atenção primária por agricultores foca-se na aquisição gratuita de medicamentos analgésicos e antitérmicos e de encaminhamento médico para a realização de exames por especialistas. Tal situação é utilizada como justificativa pelos trabalhadores da agricultura, especialmente do sexo masculino, para o acesso somente em presença de sinais e/ou sintomas de adoecimento, bem como o relato de sua invisibilidade pelos próprios profissionais da saúde e as longas distâncias a serem percorridas do domicílio aos serviços. Estas, além de requererem recursos financeiros para o deslocamento, ocasionam, por vezes, a perda de horas de trabalho e, consequentemente de produtividade. ${ }^{25}$

Tais dados contribuem para o atraso no diagnóstico e no tratamento precoce de doenças na população rural. A isso se somam elevadas taxas de subnotificações de agravos e adoecimentos, conforme identificado no estudo brasileiro sobre as notificações de câncer de pele em todo território nacional. Este foi realizado no intervalo de 2007 a 2012 e identificou 269 casos, dos quais $92 \%$ ocorreram em trabalhadores rurais, apontando incompletudes no preenchimento das notificações que promoveram uma subestimação desses dados. ${ }^{26}$

Compreende-se que a produção agrícola e sua venda exigem do trabalhador agricultor muitas horas em movimento ou na posição vertical. Fato que pode ter contribuído para a manifestação de dorsalgias nos membros superiores e inferiores. Estudo realizado no Vale do Ribeira, estado de São Paulo, identificou a presença de distúrbios osteomusculares nesses trabalhadores. As regiões mais afetadas foram a lombar, os ombros e os joelhos, que ocasionaram as maiores taxas de absenteísmo no último ano entre os trabalhadores dessa 
localidade. ${ }^{16}$ Outro estudo realizado na região centro-oeste dos Estados Unidos revelou que dor em membros superiores tem ocasionado o absenteísmo dos agricultores em aproximadamente 2,6 dias em duas semanas. ${ }^{27}$

Todavia, enfatiza-se que a negociação dos produtos agrícolas interfere, de forma positiva, na saúde do trabalhador rural. Isso porque permite o investimento em novas tecnologias que promovem a redução nos esforços físicos aplicados ao processo de trabalho. Essas evidências são demonstradas em pesquisa Asiática, que confirma esse achado à medida que revela a saúde como sinônimo de trabalho, de produção, de manter-se no lote de terra conquistado, tornando-a tecnologicamente produtiva. ${ }^{28} \mathrm{Na}$ Finlândia, o investir em modernização dos meios de produção pode ter efeitos positivos, tanto na qualidade quanto na quantidade de trabalho, bem como na segurança e saúde do trabalhador agrícola. Isso porque resulta em melhoras das condições musculoesqueléticas crônicas, as quais são associadas ao esforço físico repetitivo, às posições adotadas durante o processo de trabalho e às jornadas de trabalho extenuantes. ${ }^{29}$

No que se refere ao alcoolismo, os trabalhadores agrícolas, deste estudo percebem o vício como um hábito que pode comprometer sua saúde. Estudo australiano corrobora com esse achado à medida que revela a tendência dos agricultores em ter sua saúde física e mental comprometidas, tendo como causa base o uso de bebidas alcoólicas em grande quantidade para sanar, principalmente, o isolamento social..$^{30}$ Apesar da identificação de prejuízos à saúde decorrentes de hábitos e comportamentos não seguros, o descomprometimento dos agricultores expressa-se na busca dos serviços de saúde somente em presença de agravos e/ou adoecimento. Diferentes estudos internacionais apontam para resultados semelhantes, ao enfatizarem que os trabalhadores rurais diante de problemas comuns de saúde preferem resolvê-los sozinhos. ${ }^{7,29-30}$ Tais valores pessoais e culturais da população agrícola e a falta de preocupação com a saúde tornam-se fatores contribuintes para a dificuldade de acesso aos serviços de saúde e aumento das taxas de lesões evitáveis e doenças decorrentes de seu processo de trabalho. ${ }^{27}$ 
Riscos ocupacionais no trabalho agrícola e a negociação para a saúde do trabalhador rural | 18

Acredita-se que o não questionamento sobre a presença dos riscos ocupacionais aos trabalhadores agrícolas possa representar uma limitação desta pesquisa. No entanto, a perspectiva adotada pelas autoras objetivou apreender não somente a capacidade dos participantes do estudo em reconhecê-los, mas de refletir sobre as possibilidades de minimizálos a partir dos momentos em que compram e vendem produtos para o e do cultivo da terra.

\section{Conclusão}

Os dados obtidos revelaram que os agricultores percebem mudanças na intensidade de exposição a riscos ocupacionais decorrentes da negociação dos produtos agrícolas. Esta possibilita a eles ampliarem a jornada de trabalho no plantio e cultivo e consequentemente sua exposição direta à radiação ultravioleta emitida pelo Sol, sendo considerada um risco físico. Por outro lado, permite-os a modernização do ambiente de trabalho e a redução de esforços aplicados ao processo de trabalho, risco ergonômico.

Ao exposto, acresce-se que os trabalhadores rurais reconhecem a redução do uso de agrotóxicos em suas plantações como um efeito positivo da negociação. Além disso, percebem que essa redução impacta não somente em sua saúde, mas na das pessoas que compram seus produtos agrícolas e na saúde ambiental. Isso porque as informações provenientes do contato com revendedores e consumidores indicam aos agricultores que tal redução torna os produtos mais atrativos para a comercialização.

Tais mudanças na intensidade de exposição dos trabalhadores agrícolas a riscos ocupacionais corroboram para ampliar o lucro proveniente do processo de trabalho rural. Este representa para alguns agricultores a possibilidade de melhora e/ou recuperação de sua saúde, uma vez que lhes permite o custeio de medicamentos, de consultas médicas particulares e da assinatura de um plano de saúde para o tratamento de agravos já estabelecidos. 
Portanto, acredita-se que este estudo traz contribuições para a Enfermagem por meio da identificação de fragilidades no conhecimento dos trabalhadores rurais sobre a relação entre riscos ocupacionais e a negociação dos produtos de seu trabalho. A partir do que ela pode desenvolver ações voltadas à promoção de saúde, a exemplo das orientações de autocuidado sobre vigilância no uso de equipamentos de proteção individual, do monitoramento de condições clínicas de saúde e da frequência de acesso aos serviços de assistência à saúde, entre outros. Isso poderá permitir que os trabalhadores rurais se tornem hábeis a reconhecer comportamentos de risco no seu processo de trabalho e negociação de seus produtos e possam alterá-los para, então, melhorar suas condições de saúde. Sob essa perspectiva, o trabalhador rural torna-se sujeito ativo no que tange ao cuidado da sua saúde.

\section{Referências}

1. Deiniger K, Savastano S, Xia F. Smallholders' land access in Sub-Saharan Africa: a new landscape. Food Policy. 2017;67(2):78-92. doi: https://doi.org/10.1016/j.foodpol.2016.09.012

2. Organização das Nações Unidas para a Educação, a Ciência e a Cultura (UNESCO). Relatório mundial das nações unidas sobre desenvolvimento dos recursos hídricos 2016: água e emprego [Internet]. Brasília (DF): UNESCO; 2016 [acesso em 2020 abr 20]. Disponível em: https://arquivos.ana.gov.br/imprensa/eventosprojetos/RelatorioNacoesUnidasSobreDesenvolvimentoMun dialRecursosHidricos_2016.pdf

3. Moreira VR, Kureski R, Veiga CP. Assessment of the economic structure of Brazilian agribusiness. ScientificWorldJournal. 2016;2016(7517806):1-10. doi: https://doi.org/10.1155/2016/7517806

4. Ortega MI, Sabo S, Gallegos PA, De Zapien JEG, Zapien A, Abril GEP, et al. Agribusiness, corporate social responsibility, and health of agricultural migrant workers. Front Public Health. 2016;4(54):1-10. doi: http://doi.org/10.3389/fpubh.2016.00054

5. Szewczyk M, Pazdrowski J, Golusiński P, Dańczak-Pazdrowska A, Łuczewski Ł, Marszałek S. Basal cell carcinoma in farmers: anoccupationgroupat high risk. Int Arch Occup Environ Health. 2016;89(3):497-501. doi: https://doi.org/10.1007/s00420-015-1088-0

6. Lie A, Skogstad M, Johannessen HA, Tynes T, Mehlum IS, Nordby KC, et al. Occupational noise 
Riscos ocupacionais no trabalho agrícola e a negociação para a saúde do trabalhador rural | 20

exposure and hearing: a systematic review. Int Arch Occup Environ Health. 2016;89(3):351-72. doi: https://doi.org/10.1007/s00420-015-1083-5

7. Bhattarai D, Singh SB, Baral D, Sah RB, Budhathoki SS, Pokharel PK. Work-related injuries among farmers: a cross-sectional study from rural Nepal. J Occup Med Toxicol. 2016;11(1):48-59. doi: https://doi.org/10.1186/s12995-016-0137-2

8. Bastos RC, Bifano ACS. "Estado da Arte" sobre as publicações científicas envolvendo o trabalho agrícola familiar no Brasil sob o ponto de vista ergonômico. Eng Agric. 2017;25(1):27-37. doi: https://doi.org/10.13083/reveng.v25i1.736

9. Moreira JPL, Oliveira BLCA, Muzi CD, Cunha CLF, Brito AS, Luiz RR. A saúde dos trabalhadores da atividade rural no Brasil. Cad Saúde Pública. 2015;31(8):1698-708. doi: https://doi.org/10.1590/0102-311X00105114

10. Gesesew HA, Woldemichael K, Massa D, Mwanri L. Farmers Knowledge, Attitudes, practices and health problems associated with pesticide use in rural irrigation villages, Southwest Ethiopia. PLoS One. 2016;11(9):1-13. doi: https://doi.org/10.1371/journal.pone.0162527

11. Urio A, Souza JB, Geremia DS, Heidemann ITSB, Colliselli L. Promoção da saúde do jovem agricultor familiar: perspectiva para um envelhecimento saudável. Rev Enferm UFSM. 2020; 10(e43):1-19. doi: https://doi.org/10.5902/2179769240131

12. Osorio-Quintero L, Lopera-García LD, López-Arango YL, Rendón-Ospina ID, Tabares-López JC, Medina-Tamayo $\mathrm{M}$, et al. Condiciones de trabajo y de seguridad social en asociaciones de pequeños y medianos agricultores campesinos con prácticas de economía solidaria en tres municipios del oriente antioqueño, Colombia, 2015. Rev Fac Nac Salud Pública. 2019;37(2):36-48. doi: http://dx.doi.org/10.17533/udea.rfnsp.v37n2a05

13. Pessoa VM, Almeida MM, Carneiro FF. Como garantir o direito à saúde para as populações do campo, da floresta e das águas no Brasil? Saúde Debate. 2018;42(1):302-14. doi: https://doi.org/10.1590/0103-11042018S120

14. Cezar-Vaz MR, Bonow CA, Mello MCVA, Silva MRS. Abordagem socioambiental na enfermagem: focalizando o trabalho rural e o uso de agrotóxicos. Rev Bras Enferm. 2016;69(6):1179-87. doi: https://doi.org/10.1590/0034-7167-2016-0364

15. Oliveira JLC, Magalhães AMM, Matsuda LM. Métodos mistos na pesquisa em enfermagem: possibilidades de aplicação à luz de Creswell. Texto Contexto Enferm. 2018;27(2):e0560017. doi: https://doi.org/10.1590/0104-070720180000560017

16. Simas JMM, Alencar MCB, Yamauchi LY. Distúrbios osteomusculares em trabalhadores da bananicultura. BrJP. 2020;3(1):33-6. doi: https://doi.org/10.5935/2595-0118.20200008

17. Instituto Brasileiro de Geografia e Estatística (IBGE). Censo demográfico e contagem da população, 
2010. Tabela 1575 - Pessoas de 10 anos ou mais de idade, ocupadas na semana de referência, por seção de atividade do trabalho principal - Resultados Gerais da Amostra [Internet]. [acesso em 2020 abr 20]. Disponível em: https://sidra.ibge.gov.br/tabela/1575

18. Dancey CP, Reidy J. Estatística sem matemática para psicologia. 5ํㅡㄹ ed. Porto Alegre: Artmed; 2013.

19. Souza MAR, Wall ML, Thuler ACMC, Lowen IMV, Peres AM. The use of IRAMUTEQ software for data analysis in qualitative research. Rev Esc Enferm USP. 2018;52:e03353. doi: http://dx.doi.org/10.1590/S1980-220X2017015003353

20. Kotani K. Transportation issues in rural healthcare. J Prev Med Public Health. 2020;53(2):149-50. doi: https://doi.org/10.3961/jpmph.20.038

21. Borges AM, Bonow CA, Silva MRS, Rocha LP, Cezar-Vaz MR. Agricultura familiar e a conservação da saúde humana e ambiental. Rev Bras Enferm. 2016;69(2):326-34. doi: https://doi.org/10.1590/00347167.2016690216i

22. Khan M, Damalas CA. Occupational exposure to pesticides and resultant health problems among cotton farmers of Punjab, Pakistan. Int J Environ Health Res. 2015;25(5):581-21. doi: https://doi.org/10.1080/09603123.2014.980781

23 Silvério ACP, Martins I, Nogueira DA, Mello MAS, Loyola EAC, Graciano MMC. Assessment of Primary Health Care for rural workers exposed to pesticides. Rev Saude Publica. 2020;54:09. doi: http://dx.doi.org/10.11606/s1518-8787.2020054001455

24. Murchie P, Adam R, Khor WL, Raja EA, Iversen L, Brewster DH, et al. Impact of rurality on processes and outcomes in melanoma care: results from a whole-Scotland melanoma cohort in primary and secondary care. Br J Gen Pract. 2018;68(673):e566-75. doi: https://doi.org/10.3399/bjgp18X697901

25. Miranda SVC, Oliveira PSD, Moraes VCM, Vasconcellos LCF. Necessidades e reivindicações de homens trabalhadores rurais frente à atenção primária à saúde. Trab Educ Saúde. 2019;18(1):1-22. doi: https://doi.org/10.1590/1981-7746-sol00228

26. Cordeiro TMSC, Carneiro Neto JN, Mattos AIS, Souza FO, Merces MC, Santana TS. Câncer relacionado ao trabalho no Brasil: descrição das notificações, 2007-2012. Mundo Saúde. 2017; 41(2):23243. doi: https://doi.org/10.15343/0104-7809.20174102232243

27. Brumby SA, Ruldolphi J, Rohlman D, Donham KJ. Translating agricultural health and medicine education across the Pacific: a United States and Australian comparison study. Rural Remote Health. 2017;17(1):3931. doi: https://doi.org/10.22605/rrh3931

28. Pandey VL, Dev SM, Jayachandran U. Impact of agricultural interventions on the nutritional status in South Asia: a review. Food Policy. 2016;62:28-40. doi: https://doi.org/10.1016/j.foodpol.2016.05.002 
Riscos ocupacionais no trabalho agrícola e a negociação para a saúde do trabalhador rural | 22

29. Karttunen JP, Rautiainen RH, Lunner-Kolstrup C. Occupational health and safety of finnish dairy farmers using automatic milking systems. Front Public Health. 2016;4(147):1-11. doi: https://doi.org/10.3389/fpubh.2016.00147

30. Brew B, Inder K, Allen JAK, Thomas M, Kelly B. The health and wellbeing of Australian farmers: a longitudinal cohort study. BMC Public Health. 2016;16(988):1-11. doi: https://doi.org/10.1186/s12889-016-3664-y

Editor Científico: Tânia Solange Bosi de Souza Magnago

Editor Associado: José Luís Guedes dos Santos

Fomento/Agradecimento: Ao Conselho Nacional de Desenvolvimento Científico e Tecnológico - CNPq pelo financiamento do projeto de pesquisa intitulado "Natureza Humana da Força de Trabalho Masculina e Feminina: um estudo com trabalhadores (as) em dois ambientes rurais do Rio Grande do Sul”. Chamada MCTI/CNPq/SPM-PR/MDA n⿳⺈冂32/2012 - Processo nº. 405285/2012-4.

\section{Autor correspondente}

Leticia Silveira Cardoso

E-mail: 1sc_enf@yahoo.com.br

Endereço: Rua Júlio de Castilhos, 1934/202

CEP: 97501-753

\section{Contribuições de Autoria}

\section{1 - Leticia Silveira Cardoso}

Concepção ou desenho do estudo/pesquisa, análise e/ou interpretação dos dados

\section{2 - Clarice Alves Bonow}

Concepção ou desenho do estudo/pesquisa, análise e/ou interpretação dos dados

\section{3 - Daiani Modernel Xavier}

Revisão final com participação crítica e intelectual no manuscrito

\section{4 - Marta Regina Cezar-Vaz}

Concepção ou desenho do estudo/pesquisa, revisão final com participação crítica e intelectual no manuscrito

\section{Como citar este artigo}

Cardoso LS, Bonow CA, Xavier DM, Cezar-Vaz MR. Riscos ocupacionais no trabalho agrícola e a negociação para a saúde do trabalhador rural. Rev. Enferm. UFSM. 2021 [Acesso em: Ano Mês Dia]; vol.11 e43: 1-22. DOI: https://doi.org/10.5902/2179769248096 